\title{
Book Review: Psychophysiological Methods in Language Research - Rethinking Embodiment in Studies of Linguistic Behaviors by Bahiyyih Hardacre
}

\author{
Meagan Driver, Michigan State University \\ https://orcid.org/0000-0002-6712-4521 \\ driverme@msu.edu
}

$\mathrm{W}_{\text {ithin }}$ second language acquisition (SLA), scholars largely accept that emotion and affect are closely tied to many cognitive processes (e.g., Dewaele, 2010) and are key components driving a multitude of language learning experiences and outcomes. In the last decade, the field of language learning has seen tremendous development in research exploring emotions (e.g., Dewaele \& Li, 2020), particularly since the introduction of Positive Psychology to the field (MacIntyre et al., 2016), which has sparked additional interest in the implications of positive affective variables (e.g., Dewaele \& MacIntyre, 2014; Teimouri et al., 2020). While self-report measures are conventional in the area of language and emotions, evolving technologies have allowed scholars to explore the relationship between physiological and cognitive variables, extending the scope of research on the emotional aspects of foreign language learning. This book (Hardacre, 2020) addresses a growing curiosity in the field to implement multidimensional technologies and is the first to present an overview of the most popular psychophysiological methods, devices, and procedures for SLA scholars looking to expand their research agendas.

The book begins with a foreword by John Schumann offering a brief reflection on the relationship between second language input/output and the internal processes of the mind and how technology can be used to further investigate this connection. Next, an introduction explores the transdisciplinarity of language research and the benefits, as well as past and present challenges, of psychophysiological methods in meeting the ongoing call for multidimensional approaches in SLA (Norris \& Ortega, 2009). This is followed by ten core chapters, including one chapter on the basic principles connecting language, emotions, and the brain, and nine chapters that each present a separate psychophysiological approach to language research. 
Chapter 1 contextualizes the technical chapters that follow by summarizing the evolution of basic emotions (e.g., Ekman, 1992), the debates that exist with respect to the definition and classification of emotions (e.g., Gendron \& Barrett, 2009), and the central principles of emotion that are generally agreed upon in the field (i.e., emotions are complex, involved in physiological changes, and related to behavior and cognition). Because the chapter only briefly explores the role of emotions in language research, it requires some previous knowledge of the literature. Still, scholars looking to explore physiological approaches to research in psycholinguistics and language learning will find the summary on the relationship between emotions, the nervous system (i.e., cerebral regions, hormones), and physiological changes (e.g., skin conductance), and how these are connected to social engagement (i.e., polyvagal theory) useful for motivating further inquiry in the field.

The rest of the book is dedicated to individual chapters each dedicated to a specific psychophysiological method found in language research. Each chapter follows a standard sequence, beginning with an overview of the approach in question, including a description of key technical terms and relevant physiological theories and processes, as well as a brief summary on how each approach has been implemented within language research. Perhaps most valuable to scholars looking to consider and compare various psychophysiological approaches, the second half of each chapter presents important considerations about each methodological procedure. This includes a summary of common factors and requirements involved in measurement techniques, as well as a description of main analysis approaches, recording devices, and software platforms associated with each method. Finally, the author ends each chapter with insight on the advantages and disadvantages of each technology and offers valuable suggestions for data collection, processing, and analysis. Readers will also appreciate the authors recommendations of various open access repositories for instruments and materials (e.g., Chapter 2, PsycTESTS; Chapter 10, International Affective Picture System, IAPS).

Chapter 2 is dedicated to electrocardiography, with a detailed description of heart physiology and how to interpret electrocardiograms (ECG/EKGs). A particular emphasis is placed on heart rate variability (HRV) as a bio-marker of inhibitory and stimulating emotional states. Notably, though the author does mention general findings in electrocardiography and SLA for anxiety, she dedicates particular attention to findings on HRV and positive emotion and social behavior (e.g., empathy), which will be of use and interest to scholars in positive psychology and language learning.

Chapter 3 explores blood pressure (BP) as an index of changes in affective state. Though little information is offered on blood pressure research with respect to language, the chapter does make mention of research pertaining to stress and identity and reflects on the inverse relationship between BP, and mood and feelings of social belonging. Scholars might consider this approach to gain a wider perspective on questions around SLA and identity.

Chapters 4 and 5 review psychophysiological methods relating to skin measures. In chapter 4, electrodermal activity (EDA) is presented as one of the most commonlyused bio-signals in the field and is promoted as more accessible to many researchers thanks to its low cost, fast setup, and reliable use in even less-controlled environments. The author identifies EDA as the only psychophysiological variable that is not contaminated by parasympathetic activity and, therefore, a particularly useful tool in research on emotional and attentional processing. With respect to language research, Hardacre highlights connections between EDA and studies in discourse analysis. Chapter 5 explores skin temperature as a measure of emotional response and proposes infrared thermal imaging as a method for SLA researchers, in particular, to create comfortable and context-aware learning environments that respond to learners' body language, body temperature, and facial expressions. Language scholars interested in skinrelated psychophysiological methods might, however, consider EDA and skin temperature for a wider realm of research than presented in these chapters, in particular for questions relating to emotion, arousal, or cognitive state in SLA.

Chapter 6 presents electroencephalography as an approach that measures electrical activity in the brain through the use of electrodes placed on the scalp. The test involved, an electroencephalogram (EEG), is namely used to explore neural mechanisms associated with cognitive and emotional processing. Language researchers have conducted EEG studies to investigate neural representations relating to language production and speech comprehension, as well as cognitive control and inhibition during language 
switching, in SLA. Though not explicitly mentioned in the chapter, scholars in positive psychology and language learning may take a particular interest in this approach to explore questions around mindfulness and general wellbeing for second language learners.

In chapter 7, Hardacre summarizes functional magnetic resonance imaging (fMRI), which measures changes in cerebral blood flow in response to electrical activity fluctuations during tasks. Language scholars can implement language tasks and neuroimaging through fMRI to create brain maps for specific language processes. While fMRI collects good spatial information, it provides only an indirect measure of neural activity and, therefore, poor temporal resolution. To account for this limitation, the author recommends combining fMRI with EEG for maximal spatial and temporal recording.

Chapter 8 explores eye tracking, a measurement of eye movement activity, including eye fixations, pupil dilation, and gaze pathway patterns. Heatmaps and scan paths created through this method can act as indicators of attention and are suggested as particularly valuable in studies exploring variables related to reading. The author highlights that there are also limitations to consider when using eye tracking to register attention and discusses the benefits of complementing this approach with other brain imaging methods or with retrospective think-aloud protocols (Guan et al., 2006). Though not cited in the chapter, Godfroid's (2019) text is a valuable resource for scholars in SLA who wish to further explore eye tracking beyond the introduction offered in this book.

In chapter 9, the author presents an overview of respiration. However, she also notes that the few devices that exist for measuring respiration, in addition to the requirements for this type of method, can lead to many limitations when studying factors related to linguistic or social behavior. Still, Hardacre encourages scholars to consider respiratory data for exploring the physiological effects of mental load and stress, physiological correlates of affective variables, and particularly highlights the potential use of respiration methods for research on speech-planning and processing.
Finally, chapter 10 explores facial expression analysis, which measures non-verbal cues to investigate displays of emotion. The author highlights the importance of registering not only static facial images but also the dynamics and relative timing of facial actions in order to interpret the full meaning of these expressions within context. Unlike many other psychophysiological approaches, facial expression analysis offers a stronger possibility for social impact. Specifically, technologies that measure or convey facial expressions can increase accessibility to social cues and may be of particular interest to scholars in language and disability studies. Notably, however, Hardacre emphasizes the need that still exists for designing facial expression systems that are capable of considering diverse ethnicities, races, genders, and ages. As with many other methods, this one, too, can be combined with other monitors (e.g., EEG, EDA, pupil dilation) to increase accuracy in identifying emotional expressions and intensity.

This book presents a useful overview of psychophysiological methods and would be a valuable addition to any university or graduate program library as an introductory reference book. Scholars interested in exploring the realistic possibility of adopting a psychophysiological approach to their research will particularly benefit from the concise summaries on the advantages and limitations of each approach, including the skills required and costs associated with specific methods, devices and software, and analysis. Importantly, chapters do not review the use of each technology within the context of language research in great detail, and language scholars interested in any given approach should conduct their own review of the literature and consider a wider use of each methodology in their research than the author presents. Still, the organization of the book allows for a quick and easy initial comparison of devices and approaches, which can help scholars quickly eliminate approaches that will not meet their research or budgetary needs. This book is an invaluable resource for instigating research on emotions in SLA from transdisciplinary perspectives. 


\section{REFERENCES}

Dewaele, J.-M. (2010). Emotions in multiple languages. Palgrave Macmillan.

Dewaele, J. -M., \& Li, C. (2020). Emotions in second language acquisition: A critical review and research agenda. Foreign Language World, 196(1), 34-49.

Dewaele, J.-M., \& Macintyre, P. D. (2014). The two faces of Janus? Anxiety and enjoyment in the foreign language classroom. Studies in Second Language Learning and Teaching, 4(2), 237-274. https://doi.org/10.14746/ssllt.2014.4.2.5

Ekman, P. (1992). An argument for basic emotions. Cognition \& Emotion, 6(3-4), 169-200. https://doi.org/10.1080/02699939208411068

Gendron, M., \& Feldman Barrett, L. (2009). Reconstructing the past: A century of ideas about emotion in psychology. Emotion Review, 1(4), 316-339. https://doi.org/10/1177/1754073909338877

Godfroid, A. (2019). Eye tracking in second language acquisition and bilingualism: A research synthesis and methodological guide. Routledge.
Guan, Z., Lee, S., Cuddihy, E., \& Ramey, J. (2006). The validity of the stimulated retrospective think-aloud method as measured by eye tracking. In $\mathrm{CHI}$ '06: Proceedings of the SIGCHI conference on human factors in computing systems (pp. 1253-1262). ACM Press.

Hardacre, B. (2020). Psychophysiological methods in language research: Rethinking embodiment in studies of linguistic behaviors. Lexington Books.

MacIntyre, P., Gregersen, T., \& Mercer, S. (Eds.). (2016). Positive psychology in SLA. Multilingual Matters.

Norris, J. M., \& Ortega, L. (2009). Towards an organic approach to investigating CAF in instructed SLA: The case of complexity. Applied Linguistics, $30(4), 555-578$. https://doi.org/10.1093/applin/amp044

Teimouri, Y., Plonsky, L., \& Tabandeh, F. (2020). L2 grit: Passion and perseverance for second-language learning. Language Teaching Research. Advance online publication. https://doi.org/10.1177/1362168820921895

\section{FULL PUBLICATION DETAILS}

Title: Psychophysiological Methods in Language Research: Rethinking Embodiment in Studies of Linguistic Behaviors

Authors: Bahiyyih Hardacre

Date: 2020

Publisher: Lexington Books

Number of pages: 221

Number of chapters: 10

ISBN-13: (hbk) 978-1-4985-6673-5

ISBN-13: (ebk) 978-1-4985-6674-2 